\title{
On Using Channel Prediction in Adaptive Beamforming Systems
}

\author{
T. R. Ramya and Srikrishna Bhashyam \\ Department of Electrical Engineering, \\ Indian Institute of Technology Madras, \\ Chennai - 600 036, India. \\ Email: \{ee04d016,skrishna\}@ee.iitm.ac.in
}

\begin{abstract}
Adaptive transmission schemes based on channel state information at the transmitter have the potential to significantly improve the performance of a wireless system by matching the transmitted signal to the time varying wireless channel conditions. However, channel estimation errors and feedback delay can limit the performance gain. In this paper, the performance gain that can be obtained using channel prediction in an adaptive beamforming system with channel estimation error and feedback delay is evaluated. Channel prediction is shown to improve outage performance significantly by reducing the effect of feedback delay. Results also show that, for a given target outage performance, channel prediction allows the system to operate at much larger channel Doppler spread.
\end{abstract}

Keywords: Adaptive transmission, channel prediction, transmit beamforming, outage probability.

\section{INTRODUCTION}

The growing demand for high data rates necessitates high spectral efficiency in modern wireless communication systems. However, multipath propagation induced fading and intersymbol interference (ISI) limit the performance of a wireless communication system. Adaptive transmission schemes that use Channel State Information (CSI) at the transmitter improve performance by matching the transmitter parameters to time varying channel conditions [1],[2]. In the case of Time Division Duplex (TDD) systems, since the same frequency band is used for both transmission and reception, channel reciprocity allows us to use the channel estimates obtained during reception for transmission as well. In the case of Frequency Division Duplex (FDD) systems, CSI has to be obtained at the transmitter using a feedback channel. Feedback involves delay and due to the time varying nature of the wireless channel, the channel at the time of transmission is different from the estimated channel. Therefore, variations due to delay and channel estimation errors have to be taken into account. For an adaptive transmission system with error free delayed feedback, the feedback delay $\tau$ should be less than $\frac{0.01}{f_{d}}$, where $f_{d}$ is the Doppler spread, for negligible impact on performance [3]. Such a stringent condition is very hard to meet in practice.

In Multiple-Input Single-Output (MISO) systems with perfect CSI feedback, transmit beamforming minimizes the information outage probability [1]. The degradation due to imperfect channel estimation and feedback delay has been studied for MISO systems in [4], [5], [6]. In [4], an adaptive modulation scheme is developed in the presence of mean feedback where the channel is modeled as Gaussian with non zero mean conditioned on the feedback value. In [5], transmission strategies that maximize the average mutual information are studied in the presence of imperfect feedback. In [6], the outage probability analysis of MISO systems in the presence of delayed feedback is presented.

Channel prediction is an efficient approach to combat the degradation due to delay. The benefit of using channel prediction on adaptive rate control and power control schemes have been studied in [7]. In this paper, we evaluate the benefit of channel prediction in adaptive beamforming MISO systems. Channel prediction based on the Wiener filtering approach is used in the analysis. Significant improvement in outage probability of the adaptive beamforming system is obtained upon employing channel prediction. The maximum Doppler frequency that can be sustained by the adaptive beamforming system for different feedback delays is also evaluated. The improvement in the maximum allowable Doppler provided by channel prediction is shown.

The rest of the paper is organised as follows: Section 2 describes the system model, training model, adaptive beamforming scheme and channel prediction algorithm. Section 3 presents outage analysis of the adaptive besoming scheme upon using outdated channel estimates. Section 4 presents the outage probability analysis of the adaptive beamforming scheme when channel prediction is used. Section 5 presents the numerical results and conclusions are drawn in section 6 .

\section{SySTEM MODEL}

The system model is depicted in Fig 1. A Multiple Input Single Output (MISO) system with $N_{T}$ transmit antennas is considered. Adaptive beamforming is done at the transmitter using the predicted channel values. The channel between each transmit antenna and the receive antenna is assumed to be frequency flat, i.e., the coherence bandwidth of the channel is much larger compared to the transmit symbol bandwidth. The received signal $y(k)$ at time instant $k$ can be represented as:

$$
y(k)=\sqrt{P} \mathbf{h}^{H}(k) \mathbf{x}(k)+n(k),
$$

where $\mathbf{x}(k)$ represents the $N_{T} \times 1$ transmit vector, $P$ is the transmit power, $\mathbf{h}(k)$ is the $N_{T} \times 1$ vector of channel values, and $n(k)$ is the zero mean, unit variance additive 
white Gaussian noise. The elements of $\mathbf{h}(k)$ are assumed to be i.i.d. zero mean circularly symmetric complex Gaussian with variance $\frac{1}{2}$ per dimension.

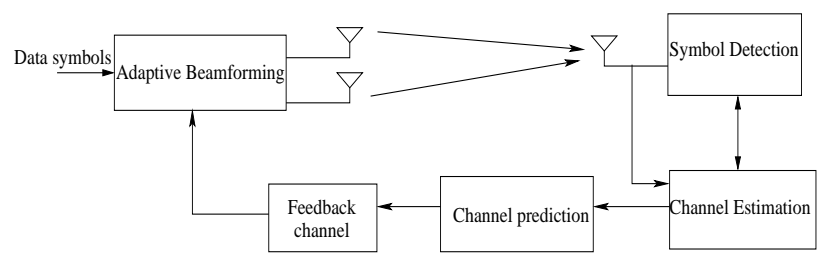

1: System Model

\section{A. Transmit Beamforming}

In MISO systems, beamforming minimizes the outage probability in the presence of perfect CSI at the transmitter [1]. The optimal beamforming vector is given by:

$$
\mathbf{b}(k)=\frac{\mathbf{h}(k)}{\sqrt{\|\mathbf{h}(k)\|^{2}}},
$$

The transmitted vector is given by:

$$
\mathbf{x}(k)=\mathbf{b}(k) d,
$$

where $d$ is the data symbol to be transmitted. Since the CSI is not perfect in practice, the estimated channel vector is used to determine the beamforming vector as follows:

$$
\mathbf{b}(k)=\frac{\tilde{\mathbf{h}}(k)}{\sqrt{\|\tilde{\mathbf{h}}(k)\|^{2}}},
$$

where $\tilde{\mathbf{h}}(k)$ is an estimate of $\mathbf{h}(k)$.

\section{B. Training Model}

Channel estimation is done at the receiver during the training phase. The estimated channel values are also fed into the channel predictor. The predicted channel values are fedback to the transmitter. The transmitter adapts the beamforming vector based on the predicted channel values.

The training pattern is depicted in Fig. 2. Every block consists of $N$ symbols out of which $N_{T}$ symbols at the beginning are used for training, one symbol for each transmit antenna. During the $i^{\text {th }}$ training instant, the $i^{\text {th }}$ transmit antenna alone is used for transmission and the corresponding channel value is estimated. Therefore, the channel value corresponding to each transmit antenna is estimated once in every $T$ seconds, where $T$ is the frame duration.

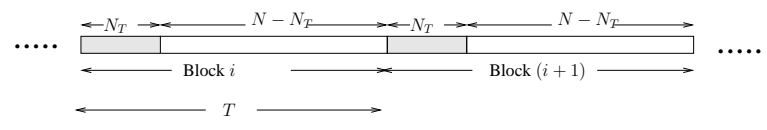

2: Training model

\section{Channel Prediction}

Channel values estimated during block $i,(i-1), \cdots,(i-$ $L+1)$ are used to predict the channel values during the block $(i+d)$, where $L$ is the prediction filter length and $d$ is the feedback delay involved. Therefore, the channel is predicted $d$ symbol durations ahead. The channel predictor is assumed to have knowledge of the delay $d$ involved and the Doppler frequency $f_{d}$. The predicted channel values are sent to the transmitter through the feedback channel.

The Wiener filtering approach is used for channel prediction. Wiener prediction is based on the Minimum Mean Square Error (MMSE) optimization criteria and exploits the time domain autocorrelation properties of the channel. The autocorrelation is modeled using the Jakes' model and is given by the zeroth order Bessel function. The channel fading corresponding to the different transmit antennas are assumed to be independent of each other. Therefore, prediction can be done separately for each transmit antenna. The prediction operation corresponding to transmit antenna $i$ is given by:

$$
\hat{h}_{i}(n)=\mathbf{w}_{i}^{H} \tilde{\mathbf{h}}_{i d},
$$

where $\hat{h}_{i}(n)$ represents the predicted channel corresponding to the transmit antenna $i$ at instant $n, \mathbf{w}_{i}$ is the vector of prediction filter weights, and

$\tilde{\mathbf{h}}_{i d}=\left[\tilde{h}_{i}(n-d), \tilde{h}_{i}(n-d-1), \ldots, \tilde{h}_{i}(n-d-(L-1))\right]^{T}$

is the vector of past channel estimates corresponding to the $i^{t h}$ transmit antenna. The optimal filter coefficients are:

$$
\mathbf{w}_{i}=\mathbf{R}_{i}^{-1} \mathbf{p}_{i}
$$

where $\mathbf{R}_{i}=E\left[\tilde{\mathbf{h}}_{i d} \tilde{\mathbf{h}}_{i d}^{H}+\sigma^{2} I_{L}\right]$ and $\mathbf{p}_{i}=E\left[\tilde{\mathbf{h}}_{i d} h_{i}(n)^{*}\right]$. The matrix $\mathbf{R}_{i}$ is the $L \times L$ autocorrelation matrix of the channel coefficients and $\mathbf{p}_{i}$ is the $L \times 1$ vector of correlation values between the past channel values and the current channel. Therefore, $\mathbf{R}_{i}(j, k)=r_{i}(k-j)+\sigma^{2} \delta(k-j)$ and $\mathbf{p}_{i}(j)=r_{i}(d+j)$. Following the Jakes model, the channel autocorrelation $r_{i}(k)$ is given by $r_{i}(k)=E\left[h_{i}(n) h_{i}(n-\right.$ $\left.k)^{*}\right]=J_{0}\left(2 \pi f_{d} T k\right)$, where $f_{d}$ is the Doppler spread. $\sigma^{2}$ represents the estimation error variance and $I_{L}$ is the identity matrix of size $L \times L$. Since all transmit antennas are assumed to have the same statistical characteristics, $\mathbf{R}_{i}=\mathbf{R}, \mathbf{p}_{i}=\mathbf{p}$, and $\mathbf{w}_{i}=\mathbf{w} \quad \forall i=1,2, \cdots, N_{T}$.

The correlation between the actual and the predicted value corresponding to the transmit antenna $i$ is defined as:

$$
\rho_{i}=\frac{E\left[h_{i}(n) \hat{h}_{i}(n)^{*}\right]}{\sqrt{E\left[\left|h_{i}(n)\right|^{2}\right] E\left[\left|\hat{h}_{i}(n)\right|^{2}\right]}}
$$

and the prediction error is given by:

$$
\epsilon_{i n}=h_{i}(n)-\hat{h}_{i}(n) .
$$

The correlation coefficient $\rho_{i}$ for the Wiener filter is given by $\rho_{i}=\sqrt{\mathbf{p}_{i}^{H} \mathbf{w}_{i}}$. Since $\mathbf{w}_{i}=\mathbf{w} \quad \forall i=1,2, \cdots, N_{T}$, $\rho_{i}=\rho \quad \forall i=1,2, \cdots, N_{T}$. The correlation coefficient $\rho$, where $0 \leq \rho \leq 1$, forms the measure of the performance of 
the filter. $\rho=1$ implies that the predicted channel is equal to the actual channel, while $\rho=0$ means that the predicted channel is independent of the actual channel. The factor $\rho$ depends on filter length, delay, Doppler spread, and $\sigma^{2}$. The dependence of $\rho$ on delay and filter length is depicted in Fig 3 . It is evident that $\rho$ increases with filter length. However, the improvement diminishes at larger filter lengths for all delays. The $j^{t h}$ tap of the filter weighs the channel estimate corresponding to the delay of $(d+j) T$. For increasing values of $j$, the past channel estimates becomes less correlated with the current channel. Therefore, increasing $L$ beyond a certain value does not improve performance. Also, as delay increases, the maximum value of $\rho$ that can be attained decreases. For a delay of $1 \mathrm{msec}$, the maximum $\rho$ that can be obtained is 0.98 , whereas for a delay of $3 \mathrm{msec}$, the maximum $\rho$ that can be obtained is only 0.8 . As delay $d$ increases, the inputs to the channel predictor are less correlated with the actual channel. Therefore, prediction performance degrades.

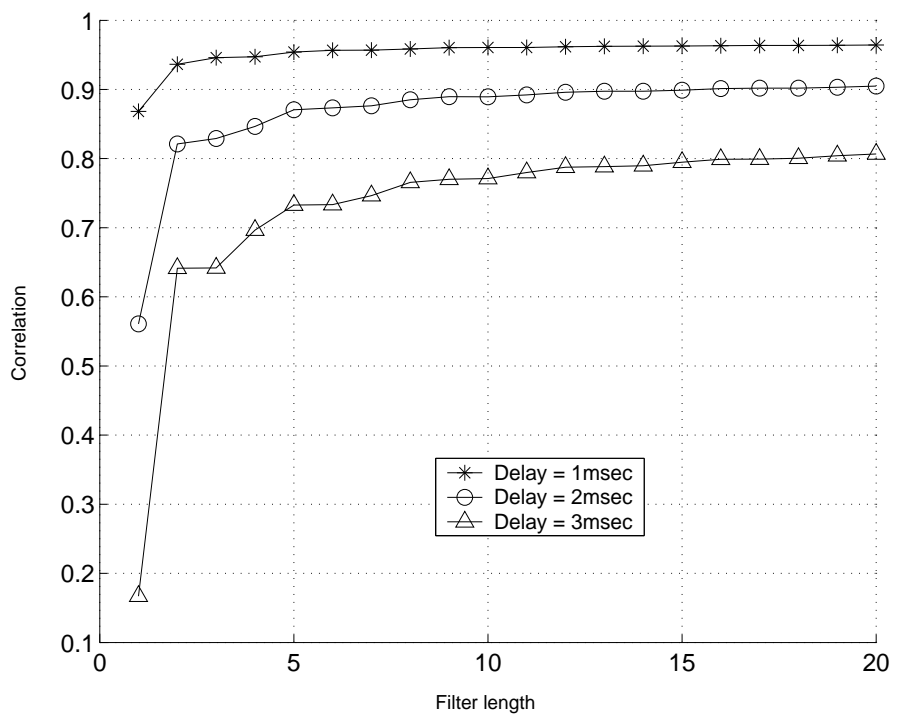

3: Correlation coeffi cient vs. Prediction filter length for different delays, Doppler frequency $=100 \mathrm{~Hz}$ and $\mathrm{SNR}=15 \mathrm{~dB}$

The relationship between the actual and the predicted channel for each antenna can be modeled (using $\rho$ ) as [6]:

$$
h_{i}(n)=\hat{h}_{i}(n)+\sqrt{1-\rho^{2}} v_{i}(n)
$$

where $v_{i}(n)$ is the zero mean unit variance Gaussian random variable independent of the channel. From this model, it is evident that given the predicted channel value $\hat{h}_{i}$, the actual channel can be modeled to be Gaussian with mean $\hat{h}_{i}$ and variance $\left(1-\rho^{2}\right)$ i.e., $h_{i}=\mathcal{N}\left(\hat{h}_{i},\left(1-\rho^{2}\right)\right) \forall i=1,2, \cdots, N_{T}$ [4]. It has been shown in [5] that for reasonably good values of $\rho$, beamforming along $\hat{h}$ is the the optimal transmission technique that maximises ergodic capacity. Similarly, in [6], it has been shown that for higher $\rho$, beamforming reduces the outage probability compared to the transmission of independent streams along the different transmit antennas.

\section{OUtAge PERFormanCE OF BEAMFORMING WITH FEEDBACK DELAY}

The delay involved in the feedback and the time varying nature of the wireless channel together lead to a different channel at the time of transmission than the channel that is fedback. Therefore, the adaptation at the transmitter is done based on the past channel values. The beamforming vector in this case is given by:

$$
\mathbf{b}=\frac{\mathbf{h}_{\text {old }}}{\sqrt{\left\|\mathbf{h}_{\text {old }}\right\|^{2}}},
$$

where $\mathbf{h}_{\text {old }}$ represents the past channel vector. The channel exhibits temporal correlations that is modeled by the Bessel function. Therefore, the current channel is related to the past channel as [6]:

$$
\mathbf{h}=\rho_{t} \mathbf{h}_{\text {old }}+\sqrt{1-\rho_{t}^{2}} v_{1}
$$

The parameter $\rho_{t}$ is given by, $\rho_{t}=J_{0}\left(2 \pi f_{d} T\right)$. The outage analysis of this system has been done in [6]. The mutual information between the transmitter and receiver for a given $\mathbf{h}$ and $\mathbf{h}_{\text {old }}$ is given by:

$$
I\left(\mathbf{x} ; y / \mathbf{h}, \mathbf{h}_{\text {old }}\right)=\log \left(1+P \mathbf{h}^{H} \mathbf{Q h}\right),
$$

where $\mathbf{Q}=E\left[\mathbf{x x}^{H}\right]$ is the transmit covariance matrix. Using equations (10) and (3), $\mathbf{Q}$ can be written as:

$$
\mathbf{Q}=\frac{\mathbf{h}_{\text {old }} \mathbf{h}_{\text {old }}^{H}}{\left\|\mathbf{h}_{\text {old }}\right\|^{2}}
$$

The outage probability is the probability that the mutual information of the system is below a given rate $R: P_{\text {out }}=$ $P(I(\mathbf{x} ; y)<R)$. Upon substituting equations (13) and (11) in equation (12), and averaging over the distribution of $\mathbf{h}$, we get [6]

$$
\begin{aligned}
P_{\text {out }}= & \frac{1}{(1+\mu)^{N_{T}-1}} \\
& \sum_{i=0}^{N_{T}-1}\left(\begin{array}{c}
M-1 \\
i
\end{array}\right) \mu^{i} \Gamma_{i+1}\left(\frac{\exp (R)-1}{P}\right),
\end{aligned}
$$

where $\mu=\frac{\rho_{t}^{2}}{1-\rho_{t}^{2}}$.

When the transmitter has perfect channel knowledge, i.e., $\rho_{t}=$ 1 , the outage probability is given by $P_{\text {out }}=\Gamma_{N_{T}}\left(\frac{\exp (R)-1}{P}\right)$. When there is no channel knowledge, $\rho_{t}=0$, the outage probability reduces to $P_{\text {out }}=\Gamma_{1}\left(\frac{\exp (R)-1}{P}\right)$.

The aim is to study the effect of channel prediction filter and the estimation error on the probability of outage. This can be done in two steps. Initially, the estimation error is modeled only in the CSI at the transmitter and the receiver is assumed to have perfect CSI. Outage probability calculated using this assumption is a lower bound on the probability of outage if the effect of estimation error is incorporated in the receiver as well. The outage probability calculations can also be done for the case of imperfect CSI at the receiver [8],[9]. In this case, an upper bound on the probability of outage can be obtained. It 
can be shown that the lower and upper bounds are close [10]. Therefore, the plot for the lower bound alone in presented in this paper.

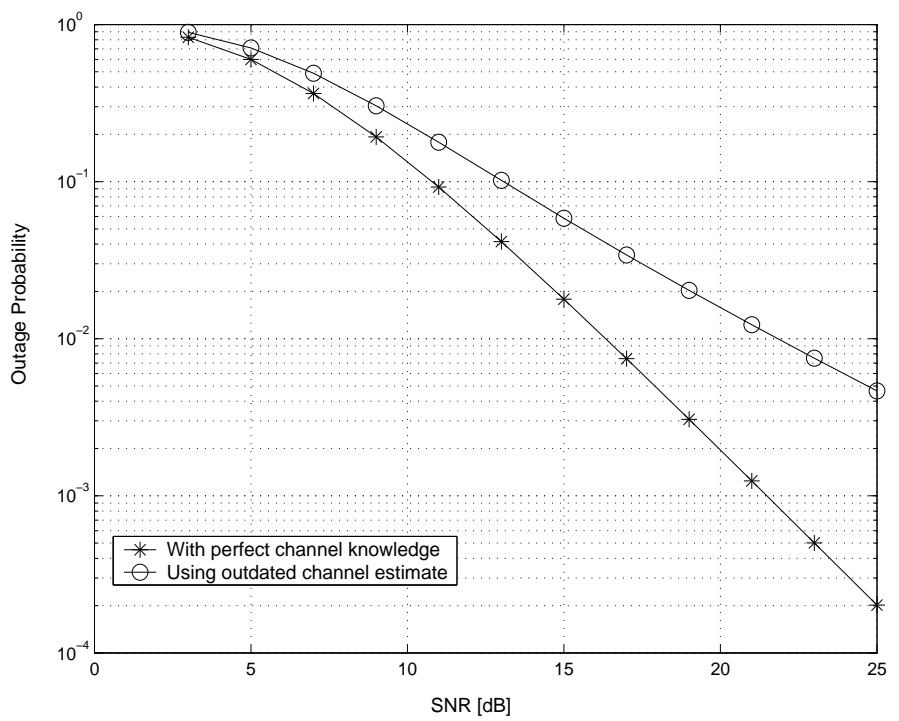

4: Outage Probability vs. SNR

The plot of the outage probability vs. SNR of the beamforming system with delayed feedback has been presented in Fig. 4 for a $N_{T}=2$ transmit antenna system. Feedback delay of $1 \mathrm{msec}$ and Doppler frequency of $100 \mathrm{~Hz}$ are considered. In this figure, the lower bound on the outage probability is presented. The outage probability of the optimal $\rho_{t}=1$ system is also plotted for the sake of comparison. It can be seen from the figure that with increasing SNR, the difference between the optimal system and the system with feedback delay increases. At an outage probability of about $10^{-2}$, the system that employs outdated estimates is about $4 \mathrm{~dB}$ away from the optimal curve.

\section{Outage Performance of Beamforming With CHANNEL PREDICTION}

The degradation due to feedback delay can be mitigated by the use of channel prediction. The predicted channel can be used for beamforming instead of the old channel vector. The beamforming vector in this case is given by:

$$
\mathbf{b}=\frac{\hat{\mathbf{h}}}{\sqrt{\|\hat{\mathbf{h}}\|^{2}}},
$$

where the $\hat{\mathbf{h}}$ is the predicted channel vector and the relation between the actual channel and the predicted channel is as given in equation (9).

The mutual information between the transmitter and receiver for a given $\mathbf{h}$ and $\hat{\mathbf{h}}$ is given by:

$$
\left.I(\mathbf{x} ; y / \mathbf{h}, \hat{\mathbf{h}})=\log \left(1+P \mathbf{h}^{H} \mathbf{Q h}\right)\right)
$$

The transmit covariance matrix $\mathbf{Q}$ is given by $\mathbf{Q}=\frac{\hat{\mathbf{h}} \hat{\mathbf{h}}^{H}}{\|\hat{\mathbf{h}}\|^{2}}$. The outage probability, $P(I(\mathbf{x} ; y)<R)$, in this case is given by:

$$
\begin{aligned}
P_{\text {pred }}= & \frac{1}{(1+\mu)^{N_{T}-1}} \\
& \sum_{i=0}^{N_{T}-1}\left(\begin{array}{c}
M-1 \\
i
\end{array}\right) \mu^{i} \Gamma_{i+1}\left(\frac{\exp (R)-1}{P}\right),
\end{aligned}
$$

where $\mu=\frac{\rho^{2}}{1-\rho^{2}}$.

The probability of outage calculation given above models the estimation error in the CSI at the transmitter only (as in section 3). It is a lower bound on the probability of outage with imperfect CSI at the receiver. An upper bound for the probability of outage can also be calculated by modeling the CSI imperfections at the receiver [10].

\section{A. Analysis of maximum allowable Doppler frequency}

For a given outage performance, the maximum Doppler frequency that can be sustained is determined as a function of the feedback delay. Consider $R=2$ nats/sec/Hz and $N_{T}=2$ transmit antennas. The probability of outage (given in equation (18)) reduces to:

$$
P_{\text {pred }}=\frac{\Gamma_{1}(\alpha)+\mu \Gamma_{2}(\alpha)}{(1+\mu)},
$$

where $\alpha=\frac{\exp (R)-1}{P}$. Suppose that we require the SNR required by the beamforming system with imperfect CSI to be within $0.5 \mathrm{~dB}$ of the SNR required by the beamforming system with perfect CSI at an outage probability of $10^{-2}$. From Fig. 4 , it can be seen that the optimal $\rho=1$ curve achieves an outage probability of $10^{-2}$ at the SNR of about $16.34 \mathrm{~dB}$. The aim is to stay within $0.5 \mathrm{~dB}$ off from the optimal curve (i.e., at $16.84 \mathrm{~dB})$. Since $P_{\text {out }}=10^{-2}, P(S N R=16.84 \mathrm{~dB})$, and $R=2$ nats $/ \mathrm{sec} / \mathrm{Hz}$ are fixed, the minimum value of $\mu$, denoted by $\mu_{t h}$ required can be calculated from equation (20). The corresponding $\rho_{t h}$ can be calculated as: $\rho_{t h}=\sqrt{\frac{\mu_{t h}}{\left(1+\mu_{t h}\right)}}$. The correlation coefficient of a Wiener filter for a given length can be calculated for different delays and Doppler frequencies. Therefore, for each delay, the maximum Doppler frequency that yields the correlation value $\rho \geq \rho_{t h}$ can be determined.

\section{Results}

A $2 \times 1$ MISO system is considered. The channel is assumed to be Rayleigh fading (zero mean unit variance complex Gaussian) with the autocorrelation modeled using the Jakes' model. The frame time $T$ is chosen to be $1 \mathrm{msec}$. Therefore, the normalised Doppler $f_{d} T=0.1$ The rate is chosen to be 2 nats/sec/Hz.

\section{A. Outage Probability vs. SNR}

The graphs of outage probability vs. SNR for the adaptive beamforming scheme (with and without channel prediction) for Doppler frequencies $50 \mathrm{~Hz}$ and $100 \mathrm{~Hz}$ are depicted in Figures 5 and 6 respectively. The performance for the prediction filter lengths of 5 and 20 are shown here. The outage probability of the system with perfect CSI at transmitter is also 
shown for the sake of comparison. The figures clearly show the improvement obtained using channel prediction. From Fig. 5 , we can see that for the Doppler ferquency of $50 \mathrm{~Hz}$, the system without prediction is about $2.5 \mathrm{~dB}$ away from the optimal curve while the system that employs prediction is within $1 \mathrm{~dB}$ from the optimal at the outage probability of $10^{-2}$. For the Doppler frequency of $100 \mathrm{~Hz}$, the system without prediction is $4 \mathrm{~dB}$ away from the optimal curve while the system with prediction is only $1 \mathrm{~dB}$ is evident from the graph that at an outage probability of about $10^{-2}$, as shown in Fig. 6. Comparing Figures 5 and 6, we can see that as Doppler frequency increases, the performance of the system without prediction degrades. This is because, with increasing Doppler, the value of $\rho_{t}=J_{0}\left(2 \pi f_{d} T\right)$ decreases. Therefore, the correlation between the past channel values (that are used for beamforming) and the current channel values decreases leading to degradation in performance. Channel prediction on the other hand, improves the correlation between the actual channel values and the CSI available at the transmitter i.e., $\rho>\rho_{t}$. Therefore the degradation due to the channel variations are reduced to a great extent.

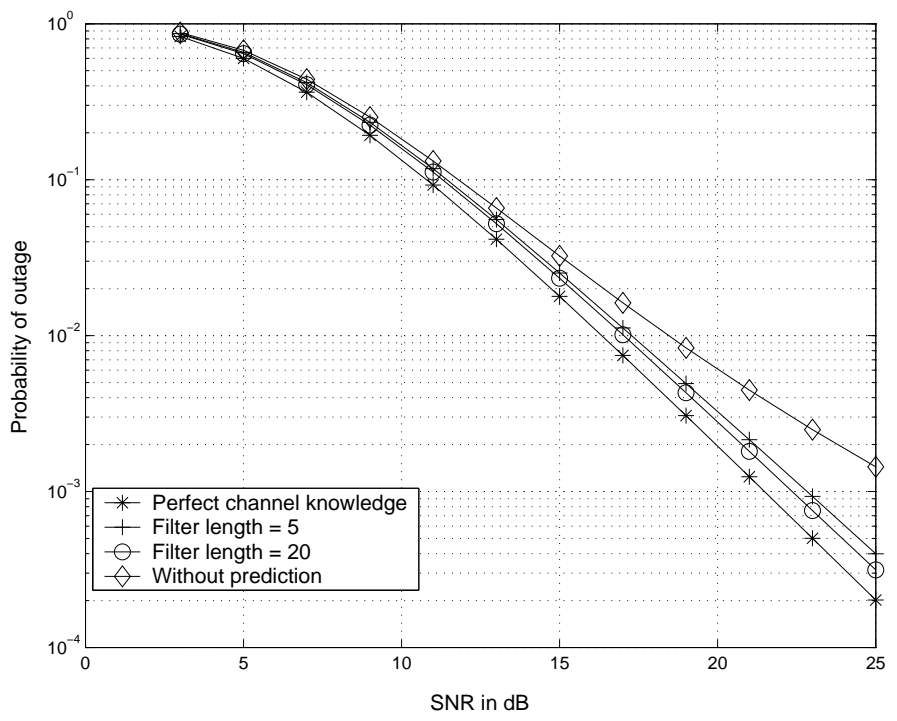

5: Outage Probability vs. SNR for Doppler frequency $=50 \mathrm{~Hz}$

\section{B. Maximum Doppler Frequency vs. Delay}

The maximum Doppler frequency vs. delay curve is shown in Fig. 7. It can be seen from the figure that for a delay of 1 msec, the system that employs channel prediction can tolerate a Doppler of $38 \mathrm{~Hz}$, while the system without prediction can withstand only $19 \mathrm{~Hz}$. Similarly for a delay of $5 \mathrm{msec}$, the system that employs prediction can withstand a Doppler of $10 \mathrm{~Hz}$, while the system with no prediction can withstand a Doppler of $5 \mathrm{~Hz}$ only. Channel prediction nearly doubles the maximum allowable Doppler frequency.

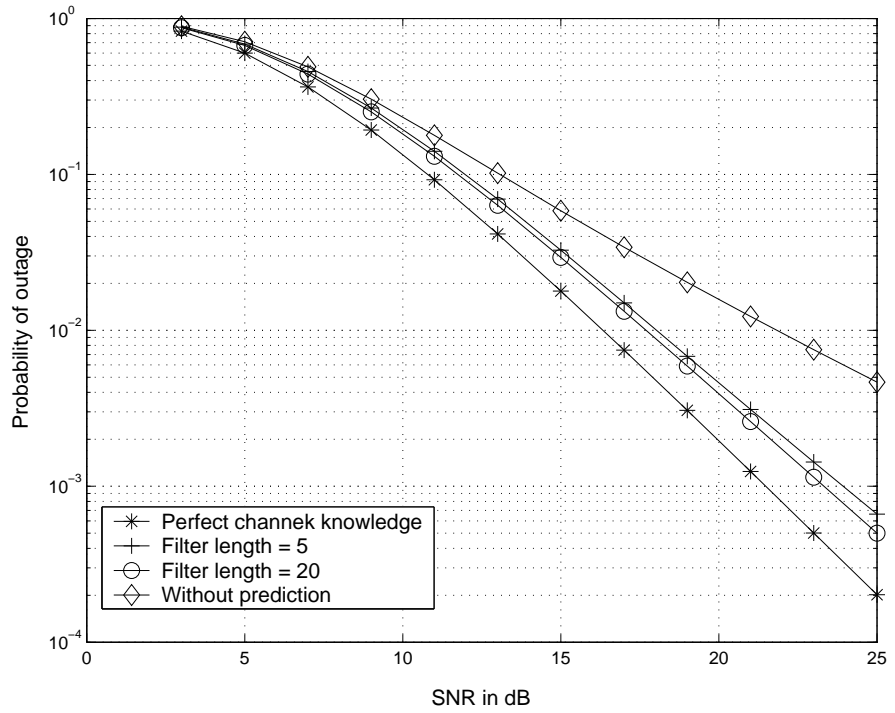

6: Outage Probability vs. SNR for Doppler frequency $=100 \mathrm{~Hz}$

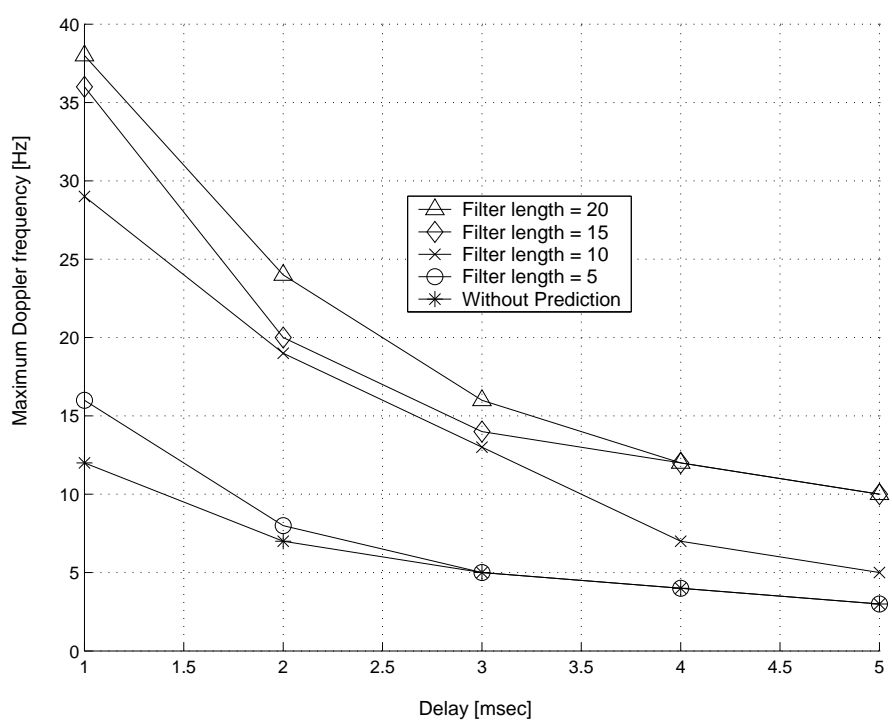

7: Maximum Doppler frequency vs. Delay for various prediction fi lter lengths

\section{CONCLUSIONS}

In this paper, the outage probability performance of an adaptive beamforming MISO system with and without channel prediction was analyzed. A channel predictor based on the Wiener filtering technique was considered in this analysis. The results show that prediction offers significant improvement in outage probability performance. For example, for a $2 \times 1$ MISO system, a $3 \mathrm{~dB}$ SNR gain is achieved at an outage probability of $10^{-2}$ for a normalized Doppler of 0.1 at 2 nats/sec/Hz. For a given outage performance, the maximum Doppler frequency that can be tolerated as a function of the feedback delay was also analyzed (with and without prediction). The tolerable Doppler spread nearly doubles upon 
using prediction.

\section{REFERENCES}

[1] E. Biglieri, G. Caire, G. Taricco, "Limiting performance of block-fading channels with multiple antennas," IEEE Transactions on Information Theory, vol. 47, no. 4, pp.1273-1289, May 2001.

[2] A. J. Goldsmith, P. P. Varaiya, "Capacity of fading channels with channel side information," IEEE Transactions on Information Theory, vol. 43, no. 6, pp. 1986-1992, Nov. 1997.

[3] M. S. Alouini, A. J. Goldsmith, "Adaptive modulation over Nakagami fading channels," Wireless Communications, vol. 13, no. 1-2, pp. 119143, May 2000.

[4] S. Zhou, G. B. Gianakkis, "Adaptive Modulation for multiantenna transmissions with channel mean feedback," IEEE Transactions on Wireless Communications, vol. 3, no. 5, pp. 1626-1636, Sep. 2004.

[5] E. Visotsky, U. Madhow, "Space Time Transmit Precoding With Imperfect Feedback," IEEE Transactions on Information Theory, vol. 47, No. 6, pp. 2632-2639, Sep. 2001.

[6] V. S. R. Annapureddy, S. Bhashyam, "Spatial and temporal power allocation for MISO systems with delayed feedback," To appear in the Proceedings of 40th Asilomar conference on Signals, Systems, and Computers, Pacifi c Grove, CA, Oct-Nov. 2006.

[7] S. Falahati, S. Svensson, T. Ekman, M. Sternad, "Adaptive modulation systems for predicted wireless channels," IEEE Transactions on Communications, vol. 52, no. 2, pp. 307-316, Feb. 2004.

[8] S. Bhashyam, A. Sabharwal, B. Aazhang, "Feedback gain in multiple antenna systems", IEEE Transactions on Communications, vol. 50, no. 5, pp.785-798, May2002.

[9] A. Lapidoth, S. Shamai, " Fading channels: How perfect need "perfect side information" be ?," IEEE Transcations on Information Theory, vol. 48, no. 5, pp. 1118-1134, May 2002.

[10] T. R. Ramya, S. Bhashyam, " Performance of adaptive multiple antenna systems with channel prediction," in preparation. 